\title{
Clinical diagnosis of malignant pleural mesothelioma
}

\author{
Andrea Bianco ${ }^{1}$, Tullio Valente ${ }^{2}$, Maria Luisa De Rimini ${ }^{3}$, Giacomo Sica $^{2}$, Alfonso Fiorelli ${ }^{4}$ \\ ${ }^{1}$ Department of Cardio-Thoracic and Respiratory Sciences, Università degli Studi della Campania "Luigi Vanvitelli”, Naples, Italy; ${ }^{2}$ Department \\ of Radiology, ${ }^{3}$ Unit of Nuclear Medicine, A.O.R.N Dei Colli, Hospital Monaldi, Naples, Italy; ${ }^{4}$ Department of Cardio-Thoracic and Respiratory \\ Sciences, Thoracic Surgery Unit, Università degli Studi della Campania "Luigi Vanvitelli", Naples, Italy \\ Contributions: (I) Conception and design: A Bianco, A Fiorelli; (II) Administrative support: A Bianco, G Sica, A Fiorelli; (III) Provision of study \\ materials or patients: A Bianco, T Valente, ML De Rimini, G Sica; (IV) Collection and assembly of data: All authors; (V) Data analysis and \\ interpretation: A Bianco, T Valente, A Fiorelli; (VI) Manuscript writing: All authors; (VII) Final approval of manuscript: All authors. \\ Correspondence to: Professor Andrea Bianco. Department of Cardio-Thoracic and Respiratory Sciences, Università degli studi della Campania "Luigi \\ Vanvitelli”, Naples, Italy; c/o Hospital Monaldi, Via L. Bianchi, 80138 Naples, Italy. Email: andrea.bianco@unicampania.it.
}

\begin{abstract}
Malignant pleural mesothelioma (MPM) is a tumour which, despite progress in diagnostic procedures and biomolecular research, has poor prognosis. Symptoms reflect extension of disease and include shortness of breath and chest pain. Unexplained pleural effusion and pleural pain in patients exposed to asbestos should raise the suspicion of MPM. MPM diagnosis requires imaging procedures X-ray and computed tomography (CT) scans; magnetic resonance imaging (MRI) better defines the extension of the tumor while PET scanning provides additional information on metabolic activity, metastases, and response to treatment. Thoracoscopic biopsy remains the most appropriate procedure for definitive diagnosis of mesothelioma. Multimodality treatment including surgery, chemotherapy and radiotherapy has been associated with a better survival in selected patients. Clinical translational research including new approaches targeting immune-checkpoints is opening new horizons which may lead to personalised treatments.
\end{abstract}

Keywords: Pleural mesothelioma; malignant pleural mesothelioma (MPM); clinical diagnosis

Submitted Aug 31, 2017. Accepted for publication Sep 26, 2017.

doi: $10.21037 /$ jtd.2017.10.09

View this article at: http://dx.doi.org/10.21037/jtd.2017.10.09

\section{Introduction}

Malignant pleural mesothelioma (MPM) is a solid tumor originating from pleural mesothelial cells. It is associated with previous asbestos exposure; delayed onset of MPM following fibre exposure (up to 40 years) makes screening for the disease challenging (1). A slight increase in incidence has been observed over the last 10 years. An estimate based on 2008 data suggested an average of 14,200 cases worldwide each year. Total incidence is highest in the United States and United Kingdom although, per capita, Australia and Italy also rank highly $(1,2)$. Most patients present at diagnosis with advanced disease; prognosis is poor with a median survival ranging from 7 to 12 months with supportive care or chemotherapy, respectively (3).

Epithelioid, sarcomatoid, and mixed or biphasic are the three major histological subtypes of MPM with sarcomatoid type exhibiting the worse prognosis (4).

In the present paper, we describe the pathogenesis, clinical features and diagnostic procedures of MPM, including imaging and invasive exams to achieve disease diagnosis.

\section{Pathogenesis}

Asbestos is the major carcinogen associated with MPM. Cancer occurs initially on the parietal surface and several pathogenetic mechanisms have been proposed (5): (I) asbestos fibres are able to irritate the pleura inducing scarring or progression to malignancy (6); (II) asbestos fibres are able to penetrate mesothelial cells, interfere with mitosis and generate mutations in DNA, altering chromosome structure (7); (III) asbestos triggers the production of oxygen free radicals responsible for intra-cellular 
DNA damage and disruption of repair mechanisms (8); (IV) asbestos is able to interfere with early-response protooncogenes which in turn promote abnormal cellular proliferation through mitogen-activated protein (MAP) kinases and extracellular signal-regulated kinases (ERK) 1 and 2 pathways (9).

Additional environmental risk factors have been implicated in MPM development such as prior radiation exposure, other mineral fibers, such as Erionite, synthetic materials (ceramics, nanoparticles), etc. $(5,10)$.

Furthermore, familial forms with autosomal dominant inheritance have been reported (5).

In addition, simian virus 40 (SV40) has also been reported as a potential cofactor in the pathogenesis of MPM, although its role remains controversial (11).

\section{Clinical presentation}

MPM symptoms are non-specific and may mimic other respiratory disease $(12,13)$. The majority of patients with MPM present with breathlessness, chest pain, weight loss and fatigue $(14,15)$.

Symptoms may be due to Ipsilateral Pleural Involvement (Parietal/Visceral), Intrathoracic Spread, TransDiaphragmatic Extension, Distant Spread, and Paraneoplastic Syndromes.

Shortness of breath is often initially due to pleural effusion; encasement caused by growing intrathoracic tumor is the major cause of breathlessness as disease progresses (16).

Thoracic pain is common and multifactorial in MPM. Tumour invasion of chest wall may cause bone pain and neuropathic pain when tumour invades neural intercostal, paravertebral or brachial plexus structures.

Symptoms associated with advanced stage of disease include weight loss, fatigue, cachexia, fevers and night sweats; at this stage thrombocytosis, hypoalbuminemia, elevated erythrocyte sedimentation rate, and anaemia $(3,5)$ are often detected.

MPM occurs initially unilaterally and local invasion of neighbouring structures including lymph node involvement may result in superior vena cava syndrome, pericardial effusion and subsequent cardiac tamponade, spinal cord compression as well as a subcutaneous involvement. The affected site becomes fixed and cannot expand. Further MPM progression may involve invasion of contralateral pleural cavity and peritoneum. Compared with lung cancer, distant metastases are usually rare since patients die before metastases occur $(1,2)$.
Only a small number of patients are asymptomatic at diagnosis, having an incidental detection of abnormality on imaging undertaken for a different reason. They appear to have longer survival than symptomatic patients, probably because they are diagnosed earlier in the disease process (1). For this reason, it is important to carefully assess patients with a background of previous asbestos exposure who present with an unexplained pleural effusion. A proportion of these patients will develop MPM over time, and active follow-up increases the chance of early diagnosis and prompt treatment.

\section{Diagnostic procedures}

Standard diagnostic work-up in patients with MPM includes Chest X-ray, computed tomography (CT) scan of chest and upper abdomen, PET, MRI to define the extension of the disease. Invasive procedures are required to obtain specimens for a definitive histological and bio-molecular diagnosis. Additional investigations include blood markers and pulmonary function tests (17-19).

\section{Chest $X$-ray}

Chest radiography (CXR) is usually the first investigation performed. Typical findings include pleural effusion with loss of hemithoracic volume and occasionally nodular pleural thickening, irregular fissural thickening or a localised mass lesion (1-4). However, it has a low sensitivity and further imaging is usually required. Figure 1 reports a case of MPM followed-up through poster anterior chest XR images from diagnosis to disease.

\section{Thoracic ultrasound (US)}

Thoracic US allows both evaluation of pleural fluid volume and detection of lesions on the pleura or diaphragm. Pleural-based mass lesions, pleural thickening $>1 \mathrm{~cm}$, nodular pleural thickening and diaphragmatic nodularity have a specificity of $>95 \%$ with regard to malignancy but, as with chest X-ray the sensitivity is low at $40 \%$ and further investigations are needed if MPM is suspected $(1,20)$.

\section{CT scan}

CT scanning at diagnosis often shows pleural effusion at disease site, pleural thickening and involvement of the interlobar fissure and invasion of the chest wall. A 

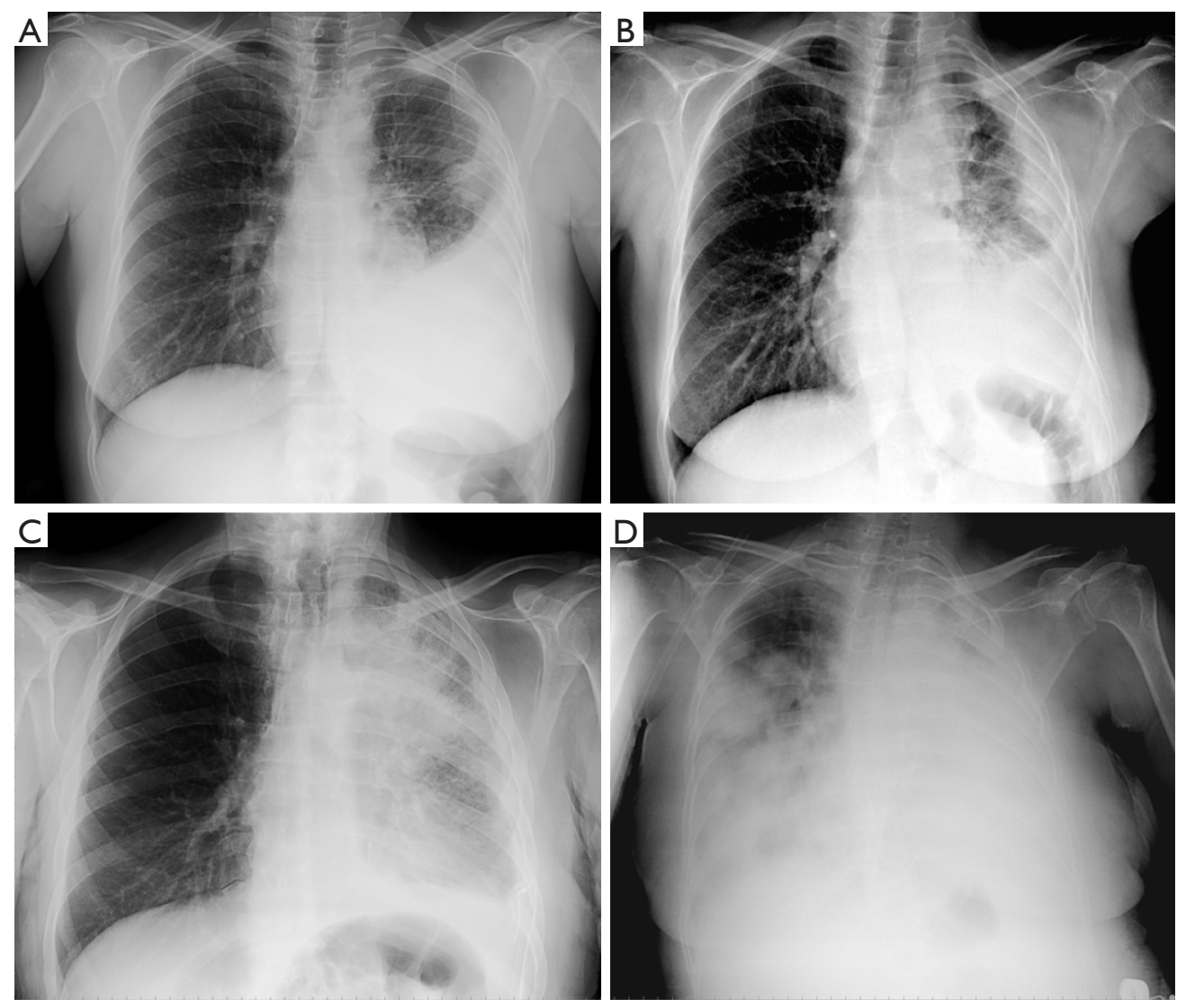

Figure 1 Poster anterior CXR follow-up images of progressive malignant pleural mesothelioma (MPM) in a 61-year-old female. (A) Baseline unilateral (left side) pleural effusion; (B) after 3 months, more diffuse pleural thickening and pleural masses; (C) after 5 months, further ipsilateral volume loss of the left hemithorax with elevation of the ipsilateral hemidiaphragm, ipsilateral mediastinal shift, and narrowing of the intercostal spaces; (D) after 8 months, contralateral spread of disease. CXR, chest radiography.

recent review of diagnostic performance of CT reported a sensitivity and specificity of $68 \%$ and $78 \%$, respectively, for pleural malignancy (21). CT cannot differentiate MPM from metastatic pleural malignancy, although circumferential pleural thickening and mediastinal pleural involvement are more frequent in MPM.

\section{Magnetic resonance imaging (MRI)}

MRI has superior soft tissue contrast over CT (20). Gadolinium contrast agent may improve delineation of T3 disease and visualize potential neoplastic foci in the diaphragm, pericardium or chest wall (22); it is particularly indicated when surgical resection is part of treatment plan.

Of the available MRI techniques, diffusion-weighted MRI (DWI), is the most promising strategy for evaluating tumor extension and response to treatment. This technique relies on tissue variability of water motility which is quantified using the apparent diffusion coefficient (ADC). Necrosis and apoptosis related to treatment decrease the tumour cell density resulting in higher water mobility and therefore an increase in ADC values; accordingly, there is a significant increase in ADC values in patients who exhibit response to treatment with chemotherapy, radiotherapy, and novel agents (23).

\section{PET-CT scan}

PET-CT scan combines high-resolution CT scanning with an injection of 18-fluoro-deoxy-glucose (FDG), which accumulates at areas of metabolic activity. FDG uptake is assessed at regions of interest and reported as standardised uptake values (SUV). Maximum SUV is higher in MPM than benign disease, and a threshold value of 2 can reliably differentiate between benign and malignant disease. 

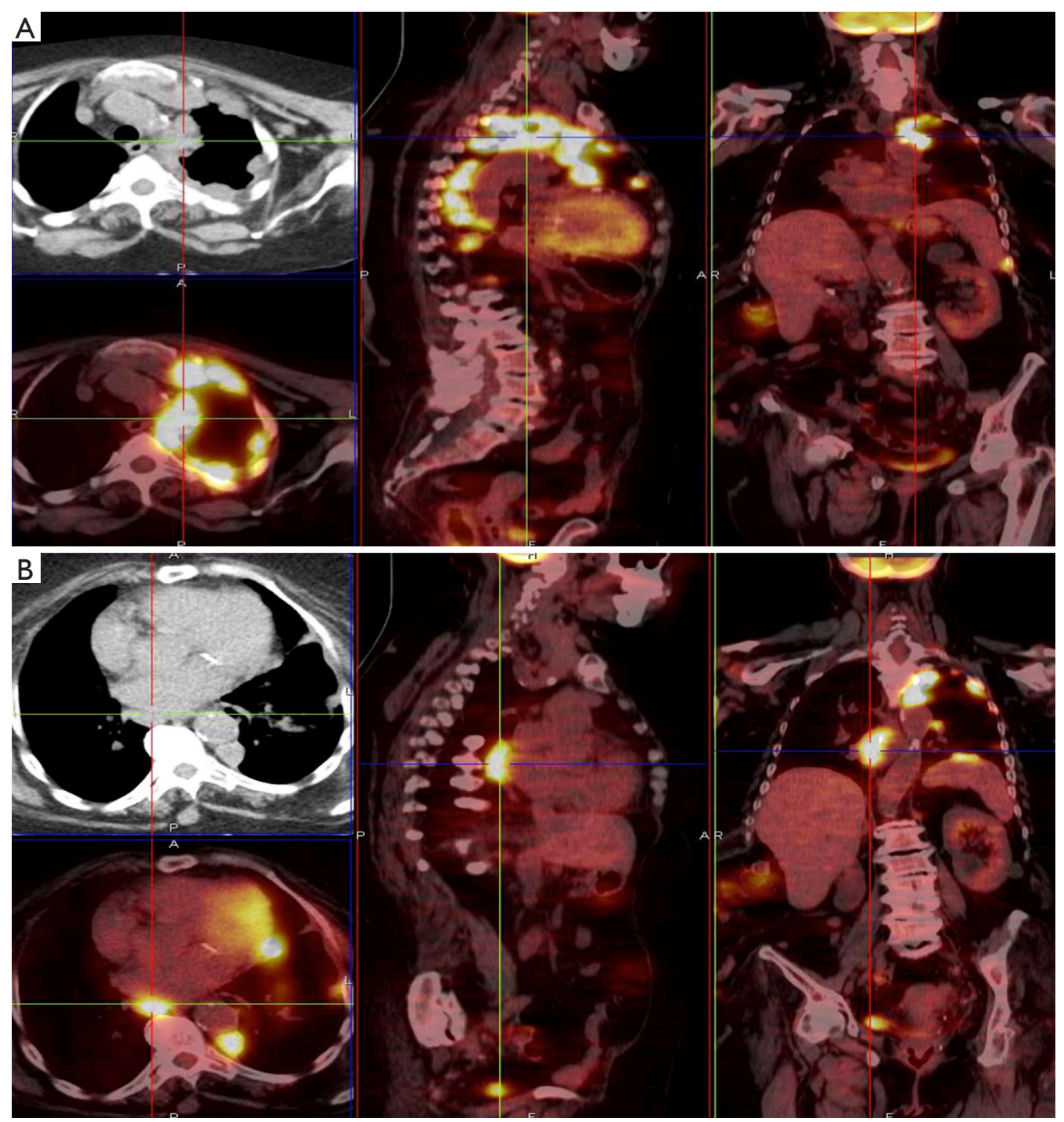

Figure 2 Whole-body ${ }^{18}$ F-FDG PET/CT. Coronal and Sagittal fusion imaging at middle and right side. Transverse reconstruction axis, at left side, has been reported as CT alone, at left top, and as fusion PET/CT imaging below: (A) MPM left unilateral side involvement; (B) severe ${ }^{18}$ F-FDG uptake of MPM pertinence at left side, with clear contralateral MPM involvement. MPM, malignant pleural mesothelioma.

Therefore PET-CT scan is employed for MPM diagnosis and staging (1) as well as to guide surgical biopsy.

PET scan has several limitations. It cannot differentiate between MPM and metastatic pleural malignancy and there is no correlation between SUV value and histological sub-type. In addition, MPM in early stage or MPM with a low proliferation rate could have a low FDG uptake (false negative results) while inflammatory disorders such as rheumatoid pleuritic, tuberculous pleurisy and prior pleurodesis could be FDG-avid (false positive results)
(1,24-27). To overcome these limits, three-dimensional measurements incorporating total tumor volume and metabolic activity may potentially be more sensitive than the SUVmax not only for diagnosis but also for evaluating the response to treatment (28).

Figure 2 reports cases of intrathoracic MPM: (I) a MPM case of unilateral intrathoracic spread; (II) a case of MPM with direct tumor invasion to the contralateral pleura. Figure 3 reports a MPM case of direct tumor invasion to the peritoneum with carcinomatosis. 

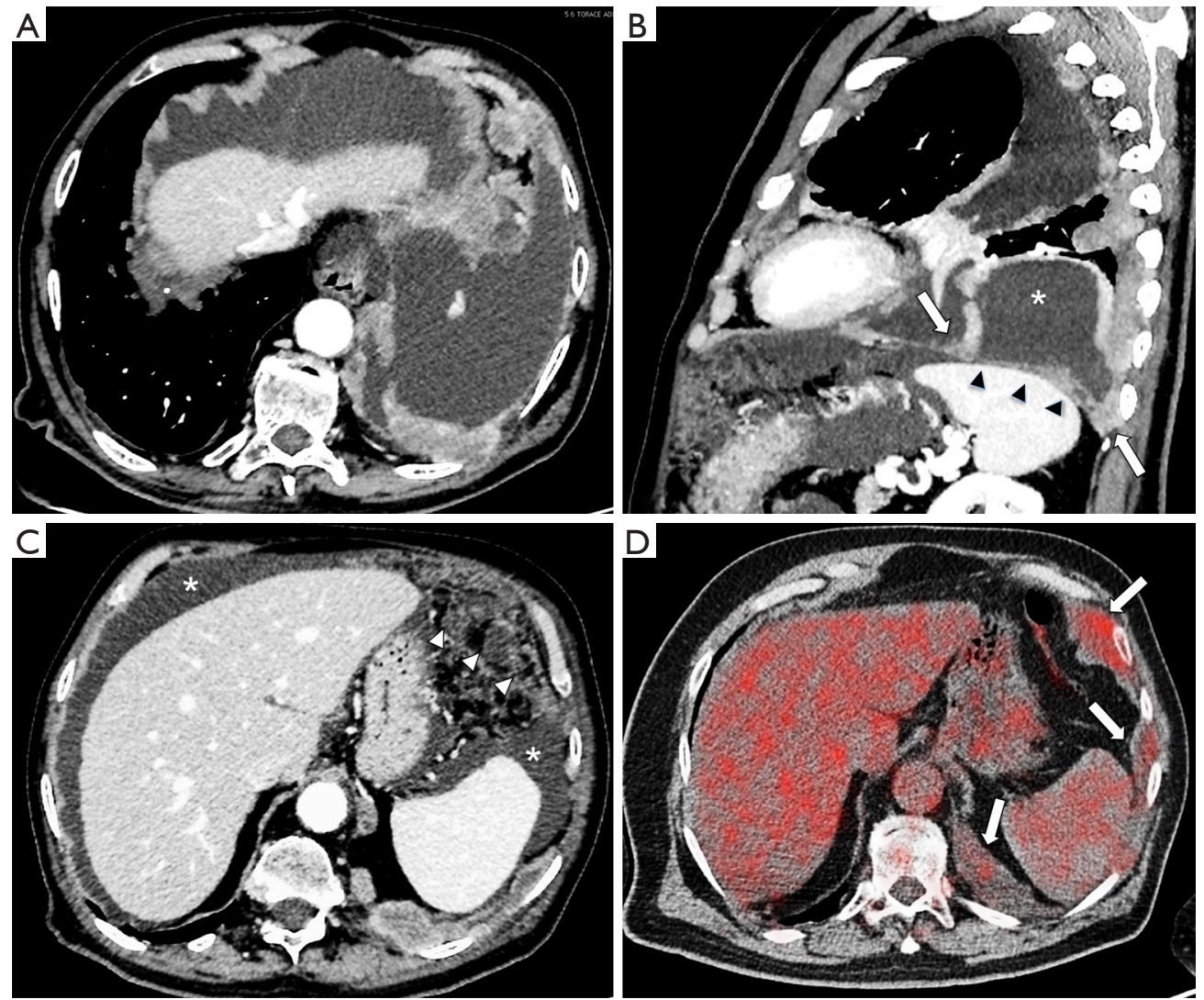

Figure 3 Trans-diaphragmatic extension in a 62-year-old man with MPM and peritoneal carcinomatosis. (A) Axial contrast enhanced wellcollimated multidetector CT (MDCT); (B) sagittal multiplanar MDCT reconstruction images at level of left hemithorax showing nodular pleural thickening in the left hemidiaphragm (arrows) and a left pleural effusion $\left(^{*}\right)$. There is complete encasement of left hemidiaphragm with loss of fat plane between diaphragm and spleen (arrowheads) suggestive of transdiaphragmatic extension; (C) MDCT image shows a thick omental thickening in the left anterior abdomen (arrowheads) and ascites $\left(^{*}\right)$ due to intraperitoneal neoplastic seeding; (D) axial fused PET/CT image at the level of superior abdomen shows FDG-avid nodular thickening in the left sub diaphragmatic region (arrows).

\section{Biomarkers}

Molecular profiling technologies to assess DNA, RNA, protein and metabolites have led to better understanding of the molecular basis of cancer, with identification of new disease markers (28-36).

Recent interest has been focused on BAP-1 protein loss (37).

Circulating serum biomarkers including mesothelin, osteopontin and vimentin have been evaluated in MPM (38).

Serum mesothelin-related protein (SMRP) has been found to be high in $84 \%$ of patient. Other serum markers such as CA-125, CA 15-3 and Hyaluronic acid are elevated while $(1,39)$, conversely, carcinoembryonic antigen (CEA) is not increased in MPM.

\section{Invasive procedures}

Prompt and accurate diagnosis of MPM is important for therapeutic and medico-legal reasons. Cytological samples obtained by thoracentesis or by fine-needle aspiration biopsy may be inconclusive for a definitive diagnosis. Large biopsies and appropriate immunohistochemistry are generally mandatory for differentiating mesothelioma from other tumors (i.e., adenocarcinoma) and for defining the histological sub-type. Biopsies can be obtained percutaneously under radiological guidance, or during thoracoscopy. In addition, VATS provides useful information on staging since it explores the entire pleural cavity, defines the extension of the tumor and detects 


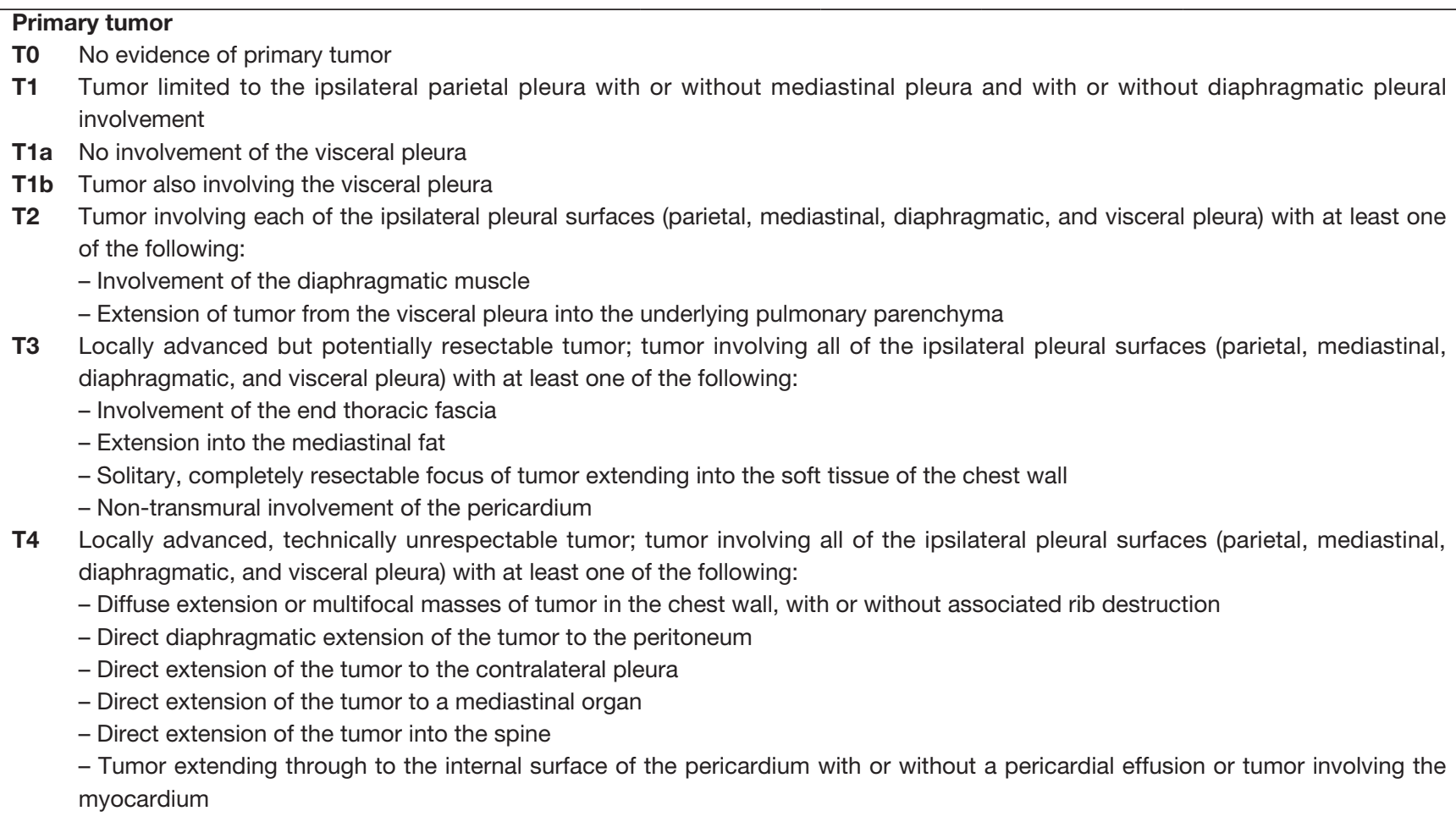

\section{Regional lymph nodes}

NX Regional lymph nodes cannot be assessed

No No regional lymph node metastases

N1 Metastases in the ipsilateral bronchopulmonary or hilar lymph node

N2 Metastases in the subcarinal or in the ipsilateral mediastinal lymph node, including the ipsilateral internal mammary and peridiaphragmatic nodes

N3 Metastases in the contralateral mediastinal, contralateral internal mammary, ipsilateral, or contralateral supraclavicular lymph nodes

\section{Distant metastases}

Mo No distant metastasis

M1 Distant metastasis

\begin{tabular}{|c|c|c|c|}
\hline \multicolumn{4}{|c|}{ Stage grouping } \\
\hline Stage & T & N & M \\
\hline IA & T1a & N0 & M0 \\
\hline IB & T1b & N0 & M0 \\
\hline II & T2 & No & M0 \\
\hline \multirow{3}{*}{ III } & T1, T2 & N1 & M0 \\
\cline { 2 - 4 } & T1, T2 & N2 & M0 \\
\cline { 2 - 4 } & T3 & N0, N1, N2 & M0 \\
\hline \multirow{3}{*}{ IV } & T4 & Any N & M0 \\
\cline { 2 - 4 } & Any T & N3 & M0 \\
\cline { 2 - 4 } & Any T & Any N & M1 \\
\hline
\end{tabular}

Figure 4 Staging for malignant pleural mesothelioma $(40,41)$.

the invasion of neighbouring structures (1-4). When imaging is equivocal, additional invasive procedures should be performed to investigate mediastinal involvement (mediastinoscopy, anterior mediastinotomy, EBUS-TBNA) and/or of contralateral pleural or peritoneum invasion (bilateral thoracoscopy and laparoscopy).

\section{Staging and response assessment}

Both staging and response assessment in MPM are difficult to be performed due to pattern of growth and disease progression. The absence of bi-dimensional measurable consolidations limits tumour size and nodal involvement assessment by conventional size-based imaging techniques. Several staging classifications have been proposed over the years, all of which have limitations for use in routine clinical practice regarding tumour (T-) and node (N-) extension. Mainly used TNM staging system was proposed by the International Mesothelioma Interest Group (IMIG) with the approval of Union for International Cancer Control (Figure 4) (40,41). 
Functional or molecular imaging techniques that reflect specific features of tumour pathophysiology, like PET, MRI -DWI and dynamic contrast-enhanced MRI (DCE-MRI) are attracting growing interest. However, despite accuracy limits, size measurement based response criteria remains widely used in MPM management $(42,43)$.

\section{New strategies}

Multimodality therapy including surgery, radiotherapy and chemotherapy remains the approach for MPM treatment (1-4). Inter-individual differences in terms of response to multimodality treatment and impaired functional status make treatment decisions challenging in MPM.

Personalised treatment based on individual patient characteristics and tumour genetics are under investigation (44).

Targeted therapies to epidermal growth factor receptor (EGFR) antagonists and platelet-derived growth factor receptor inhibitors have shown promising results. Immune checkpoint inhibitors (ICI) are attracting much interest as first-, second- or third-line treatments demonstrating prolonged disease stability. The combination of chemotherapy with ICI, or multiple ICI, has shown to be synergistic in other tumours and warrants further exploration in MPM.

\section{Conclusions}

MPM is an aggressive tumor, usually diagnosed in advanced stage and associated with a poor prognosis (ranging from 7 to 12 months). Unexplained pleural effusion and pleural pain in patients with asbestos exposure should raise the suspicion of MPM. In addition to conventional imaging, functional imaging techniques have the potential to improve disease management.

Thoracoscopic biopsy remains the most appropriate procedure for definitive diagnosis.

Multimodality approach with surgery, radiotherapy and chemotherapy is currently the standard while new personalised treatments, including ICI have shown promising results.

\section{Acknowledgements}

None.

\section{Footnote}

Conflicts of Interest: The authors have no conflicts of interest to declare.

\section{References}

1. Bibby AC, Tsim S, Kanellakis N, et al. Malignant pleural mesothelioma: an update on investigation, diagnosis and treatment. Eur Respir Rev 2016;25:472-86.

2. Baas P, Fennell D, Kerr KM, et al. Malignant pleural mesothelioma. ESMO Clinical Practice Guidelines for diagnosis, treatment and follow-up. Ann Oncol 2015;26:v31-9.

3. Geltner C, Errhalt P, Baumgartner B, et al. Management of malignant pleural mesothelioma - part 1: epidemiology, diagnosis, and staging: Consensus of the Austrian Mesothelioma Interest Group (AMIG). Wien Klin Wochenschr 2016;128:611-7.

4. Fiorelli A, Pentimalli F, D'Urso V, et al. Antineoplastic activity of povidone-iodine on different mesothelioma cell lines: results of in vitro study. Eur J Cardiothorac Surg 2014;45:993-1000.

5. Robinson BW, Lake RA. Advances in malignant mesothelioma. N Engl J Med 2005;353:1591-603.

6. Sebastien P, Janson X, Gaudichet A, et al. Asbestos retention in human respiratory tissues: comparative measurements in lung parenchyma and in parietal pleura. IARC Sci Publ 1980;30:237-46.

7. Ault JG, Cole RW, Jensen CG, et al. Behavior of crocidolite crocidolite asbestos during mitosis in living vertebrate lung epithelial cells Cancer Res 1995;55:792-8.

8. Kamp DW, Israbian VA, Preusen SE, et al. Asbestos causes DNA strand breaks in cultured pulmonary epithelial cells: role of iron-catalyzed free radicals. Am J Physiol 1995;268:L471-80.

9. Zanella CL, Posada J, Tritton TR, et al. Asbestos causes stimulation of the extracellular signal-regulated kinase 1 mitogen- activated protein kinase cascade after phosphorylation of the epidermal growth factor receptor. Cancer Res 1996;56:5334-8.

10. Mazzarella G, Lucariello A, Bianco A, et al. Exposure to submicron particles (PM1.0) from diesel exhaust and pollen allergens of human lung epithelial cells induces morphological changes of mitochondria tonifilaments and rough endoplasmic reticulum. In Vivo 2014;28:557-61.

11. Vilchez RA, Butel JS. Emergent human pathogen simian virus 40 and its role in cancer. Clin Microbiol Rev 2004;17:495-508.

12. Kim KC, Vo HP. Localized malignant pleural sarcomatoid mesothelioma misdiagnosed as benign localized fibrous tumor. J Thorac Dis 2016;8:E379-84.

13. Perrotta F, Cerqua FS, Cammarata A, et al. Integrated 
therapeutic approach to giant solitary fibrous tumor of the pleura: report of a case and review of the literature. Open Med (Wars) 2016;11:220-5.

14. Scherpereel A, Astoul P, Baas P, et al. Guidelines of the European Respiratory Society and the European Society of Thoracic Surgeons for the management of malignant pleural mesothelioma. Eur Respir J 2010;35:479-95.

15. British Thoracic Society Standards of Care Committee. BTS statement on malignant mesothelioma in the UK, 2007. Thorax 2007;62:ii1-ii19.

16. Rudd RM. Malignant mesothelioma. Br Med Bull 2010;93:105-23.

17. Labby ZE, Armato SG 3rd, Dignam JJ, et al. Lung volume measurements as a surrogate marker for patient response in malignant pleural mesothelioma. J Thorac Oncol 2013;8:478-86.

18. Maniscalco M, Bianco A, Mazzarella G, et al. Recent Advances on Nitric Oxide in the Upper Airways. Curr Med Chem 2016;23:2736-45.

19. Maniscalco M, Vitale C, Vatrella A, et al. Fractional exhaled nitric oxide-measuring devices: technology update. Med Devices (Auckl) 2016;9:151-60.

20. Yamamuro M, Gerbaudo VH, Gill RR, et al. Morphologic and functional imaging of malignant pleural mesothelioma. Eur J Radiol 2007;64:356-66.

21. Nickell LT Jr, Lichtenberger JP 3rd, Khorashadi L, et al. Multimodality imaging for characterization, classification, and staging of malignant pleural mesothelioma. Radiographics 2014; 34:1692-706.

22. Giesel FL, Bischoff H, von Tengg-Kobligk H, et al. Dynamic contrast-enhanced MRI of malignant pleural mesothelioma: a feasibility study of noninvasive assessment, therapeutic follow-up, and possible predictor of improved outcome. Chest 2006;129:1570-6.

23. Kharuzhyk S, Fabel M, von Tengg-Kobligk H, et al. Image-based evaluation of tumor response to treatment: where is radiology today? Exp Oncol 2008;30:181-9.

24. Fiorelli A, Vicidomini G, Laperuta P, et al. The role of Tc-99m-2-Methoxy-Isobutyl-Isonitrile Single Photon Emission Computed Tomography in visualizing anterior mediastinal tumor and differentiating histologic type of thymoma. Eur J Cardiothorac Surg 2011;40:136-42.

25. Brunese L, Greco B, Setola FR, et al. Non-small cell lung cancer evaluated with quantitative contrast-enhanced CT and PET-CT: net enhancement and standardized uptake values are related to tumour size and histology. Med Sci Monit 2013;19:95-101.

26. Del Giudice G, Bianco A, Cennamo A, et al.
Lung and Nodal Involvement in Non tuberculous Mycobacterial Disease: PET/CT Role. Biomed Res Int 2015;2015:353202.

27. Bianco A, Mazzarella G, Rocco D, et al. FDG/PET uptake in asymptomatic multilobar Chlamydia pneumoniae pneumonia. Med Sci Monit 2010;16:CS67-70.

28. Kitajima K, Doi H, Kuribayashi K, et al. Prognostic value of pretreatment volume-based quantitative (18)F-FDG PET/CT parameters in patients with malignant pleural mesothelioma. Eur J Radiol 2017;86:176-83.

29. Bruno R, Alì G, Giannini R, et al. Malignant pleural mesothelioma and mesothelial hyperplasia: A new molecular tool for the differential diagnosis. Oncotarget 2017;8:2758-70.

30. Cattaneo F, Guerra G, Parisi M, et al. Expression of Formylpeptide Receptors in Human Lung Carcinoma. Anticancer Res 2015;35:2769-74.

31. Wu JY, Vlastos AT, Pelte MF, et al. Aberrant expression of BARD1 in breast and ovarian cancers with poor prognosis. Int J Cancer 2006;118:1215-26.

32. Zhang YQ, Bianco A, Malkinson AM, et al. BARD1: An independent predictor of survival in non-small cell lung cancer. Int J Cancer 2012;131:83-94.

33. Pilyugin M, Descloux P, André PA, et al. BARD1 serum autoantibodies for the detection of lung cancer. PLoS One 2017;12:e0182356.

34. Illiano M, Nigro E, Sapio L, et al. Adiponectin downregulates CREB and inhibits proliferation of A549 lung cancer cells. Pulm Pharmacol Ther 2017;45:114-20.

35. Nigro E, Imperlini E, Scudiero O, et al. Differentially expressed and activated proteins associated with non small cell lung cancer tissues. Respir Res 2015;16:74.

36. Bueno R, Stawiski EW, Goldstein LD, et al. Comprehensive genomic analysis of malignant pleural mesothelioma identifies recurrent mutations, gene fusions and splicing alterations. Nat Genet 2016;48:407-16.

37. Righi L, Duregon E, Vatrano S, et al. BRCA1-Associated Protein 1 (BAP1) Immunohistochemical Expression as a Diagnostic Tool in Malignant Pleural Mesothelioma Classification: A Large Retrospective Study. J Thorac Oncol 2016;11:2006-17.

38. Bonotti A, Simonini S, Pantani E, et al. Serum mesothelin, osteopontin and vimentin: useful markers for clinical monitoring of malignant pleural mesothelioma. Int J Biol Markers 2017;32:e126-e131.

39. Fiorelli A, Morgillo F, Fasano M, et al. The value of matrix metalloproteinase-9 and vascular endothelial growth factor receptor 1 pathway in diagnosing 
indeterminate pleural effusion. Interact Cardiovasc Thorac Surg 2013;16:263-9.

40. Rusch VW, Giroux D. Do we need a revised staging system for malignant pleural mesothelioma? Analysis of the IASLC database. Ann Cardiothorac Surg 2012;1:438-48.

41. Edge S, Byrd DR, Compton CC, et al. AJCC Cancer Staging Manual. 7th edition. New York: Springer, 2010.

42. Byrne MJ, Nowak AK. Modified RECIST criteria for

Cite this article as: Bianco A, Valente T, De Rimini ML, Sica G, Fiorelli A. Clinical diagnosis of malignant pleural mesothelioma. J Thorac Dis 2018;10(Suppl 2):S253-S261. doi: 10.21037/jtd.2017.10.09 assessment of response in malignant pleural mesothelioma, Ann Oncol 2004;15:257-60.

43. Cheng L, Tunariu N, Collins DJ, et al. Response evaluation in mesothelioma: Beyond RECIST. Lung Cancer 2015;90:433-41.

44. Wald O, Sugarbaker DJ. Malignant pleural mesothelioma: key determinants in tailoring the right treatment for the right patient. J Thorac Dis 2017;9:485-9. 